\title{
Les Plécoptères de la vallée supérieure du Pô (Alpes Cottiennes). Notes faunistiques et écologiques ${ }^{1}$
}

\author{
E. Ravizza Dematteiș² \\ C. Ravizza ${ }^{3}$
}

Mots clés : Plecoptera, faunistique, écologie, plécoptérocénoses, Alpes occidentales.

55 espèces de Plécoptères ont été recensées dans la val lée supérieure du Pô (Alpes Cottiennes, Piémont, Italie) où, pendant plusieurs années, nous avons prospecté méthodiquement 7 stations lotiques situées entre 2.020 et $550 \mathrm{~m}$ d'altitude.

Après avoir indiqué brièvement les caractéristiques de la vallée du fleuve Pô et des stations d'échantillonnage, les répartitions altitudinale, saisonnière et géographique des espèces récoltées sont analysées. Les principales plécoptérocénoses rhéophiles sont déterminées et analysées en fonction des paramètres des biotopes et des données qualitatives et semiquantitatives.

Running-water Stoneflies of the high Po valley (Cottian Alps). Faunistic and ecologieal notes.

Keywords : Plecoptera, faunistic, ecology, stonefly-communities, Western Alps.

55 species of Plecoptera were recorded in the Po val]ey (Cottian Alps, Piedmont, Italy), where 7 running-water stations, between 2,020 and $550 \mathrm{~m}$ a.s.l., were methodically investigated for many years.

After a brief description of the characteristics both of the valley and of the Po river, as well as of the sampling stations, the species collected in each of them are listed : the attitudinal, seasonal and geographical distribution of the stoneflies are discussed. With reference to both the biotope parameters and the qualitative and semiquantitative data, the main stonefly-communities are identified and discussed.

\section{Introduction}

Bien que le Pô soit le fleuve le plus important d'Italie, la faune de Plécoptères de son cours de montagne était encore presque inconnue. Dans le but de combler cette lacune, nous avons entrepris des recherches qui se sont déroulées pendant plus de dix ans et qui ont été plus activement menées entre 1982 et 1985. En vue de recenser la faune de Plécoptères et d'étudier les communautés rhéophiles, nous avons prospecté méthodiquement 5 stations situées

1. Recherches effectués à l'aide d'une subvention partielle du M.E.N. Italien (M.P.I. $60 \%$ ).

2. Dipartimento di Biologia dell'Università, sezione ecotogia, Via Celoria 26, I-20133 Milano, Italie.

3. Largo O. Murani 4. 1-20133 Mitano. Italie. sur le cours supérieur du Pồ, depuis la source à $2.020 \mathrm{~m}$, jusqu'à la vallée vers $550 \mathrm{~m}$, soit sur un parcours d'environ $20 \mathrm{~km}$. Deux autres stations d'échantillonnage ont été choisies surdes affluents de montagne. Dans ces 7 stations (fig. 1), représentant les biotopes les plus caractéristiques de la haute vallée, nous avons recensé en tout 55 espèces 1 .

\section{Le cours supérieur du Pô}

Le Pô prend sa source vers $2.020 \mathrm{~m}$ dans le Piano del $\mathrm{Re}$, petite plaine alluviale sur le versant nord

1. Dans deux notes se rapportant aux premières années de recherche nous avions dressé une liste préliminaire de 48 espèces (Ravizza Dematteis \& Ravizza 1983b) et décrit une espèce nouvelle (Ravizza \& Ravizza Dematteis 1984). 


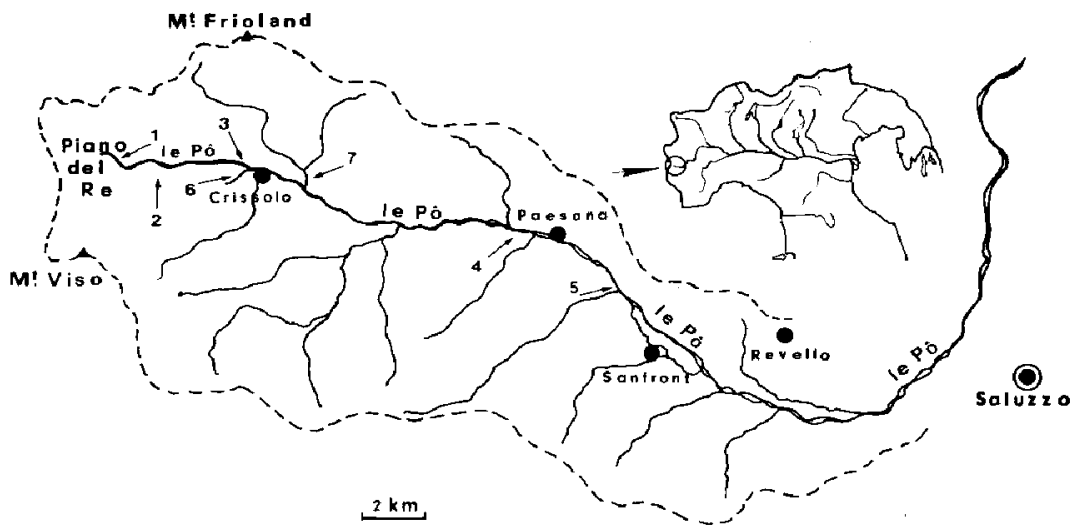

Fig. 1. Carte de la vallée supérieure du Pô et des stations étudiées

du mont Viso, et se jette dans la mer Adriatique après un parcours d'environ $652 \mathrm{~km}$. De sa source à son débouché dans la plaine vers $330 \mathrm{~m}$ près de Revello, le Pô coule sur une distance de $35 \mathrm{~km}$, ce qui correspond à une dénivellation d'environ 1.700 $m$ et à une pente moyenne de 4,86\% (Gribaudi 1966).

Le régime du cours de montagne du Pô est de type nivo-pluvial, avec deux périodes de hautes eaux : l'une plus prononcées de mai au début de juillet lors de la fonte des neiges et des glaciers, l'autre plus faible en automne, et deux étiages: l'un en été et l'autre en hiver.

Il n'existe qu'une station de mesure du débit sur ce tronçon, située à Crissolo, où le débit moyen du Pô est de $1,49 \mathrm{~m}^{3} / \mathrm{s}$ (IRSA 1977). Plus en aval, vers 700 $\mathrm{m}$, un barrage détourne une partie des eaux et par conséquent au-dessous le débit du Pô est comparable à celui de la rivière vers $1.700 \mathrm{~m}$. En aval de Sanfront, les eaux du Pô s'infiltrent dans les graviers d'un imposant cône de déjection et, sur une longueur de $6 \mathrm{~km}$, jusqu'en aval de Revello, le fleuve n'occupe toute la largeur de son lit que lors des crues saisonnières (Gribaudi 1966); il se réduit donc à un ruisseau pendant la plus grande partie de l'année.
Les précipitations atmosphériques annuelles sont en moyenne de $1.100 \mathrm{~mm}$ dans toute la vallée. Pendant l'été on observe souvent des brumes surtout en altitude où des orages éclatent fréquemment pendant l'après-midi. En hiver la couche de neige est abondante, notamment au-dessus de Crissolo.

Du point de vue lithologique, le mont Viso et les autres pics fort escarpés qui l'entourent sont constitués de roches métamorphiques (roches vertes). Plus bas, les pentes moins abruptes correspondent à des dépôts détritiques et morainiques qui recou. vrent un socle de schistes lusirés. En dessous de Crissolo, jusque vers $700 \mathrm{~m}$, la vallée creusée dans les gneiss et les schistes lustrés, est très étroite et encaissée. Plus en aval, vers Paesana, prédominent des roches plus tendres: micaschistes et schistes graphitiques ; la vallée s'élargit et la pente diminue.

\section{Méthodes d'échantillonnage et techni- ques d'analyse des eaux}

Dans toutes les stations les echantillonnages ont éte effectués sur des surfaces à peu près identiques pendant une heure. 
Tableau I. Caractéristiques de l'eau des stations

\begin{tabular}{|c|c|c|c|c|c|c|c|}
\hline station $n^{\circ}$ & 1 & 2 & 3 & 4 & 5 & 6 & 7 \\
\hline altitude m & 2.020 & 1.700 & 1.380 & 650 & 550 & 1.380 & 1. 150 \\
\hline nombre de mesures & $\mathfrak{f 5}$ & 21 & 28 & 20 & 14 & 28 & 24 \\
\hline période des relevés & $\mathrm{v}-\mathrm{x}$ & I I I -XI & I-XII I & $I-X I_{I} I$ & I-XI I & $I-X I I$ & $I-X I I$ \\
\hline temp. ${ }^{\circ} \mathrm{C} \frac{\min }{\max }$ & $\begin{array}{l}2,0 \\
7,0\end{array}$ & $\begin{array}{r}3,0 \\
10,5\end{array}$ & $\begin{array}{r}2,0 \\
11,5\end{array}$ & $\begin{array}{r}2,0 \\
15,0\end{array}$ & $\begin{array}{r}3,0 \\
19,0\end{array}$ & $\begin{array}{l}4,5 \\
7,0\end{array}$ & $\begin{array}{r}1,5 \\
13,0\end{array}$ \\
\hline $\mathrm{pH}_{\max }^{\min }$ & $\begin{array}{l}6,5 \\
7,0\end{array}$ & $\begin{array}{l}7,0 \\
7,0\end{array}$ & $\begin{array}{l}7,0 \\
7,5\end{array}$ & $\begin{array}{l}7,0 \\
7,5\end{array}$ & $\begin{array}{l}7,0 \\
7,5\end{array}$ & $\begin{array}{l}7,0 \\
7,5\end{array}$ & $\begin{array}{l}7,0 \\
7,0\end{array}$ \\
\hline $\begin{array}{ll}\text { dureté tot, } & \text { min } \\
\mathrm{ppm} \mathrm{CaCO}_{3} & \text { max }\end{array}$ & $\begin{array}{l}35,60 \\
53,40\end{array}$ & $\begin{array}{l}35,60 \\
53,40\end{array}$ & $\begin{array}{l}53,40 \\
89,00\end{array}$ & $\begin{array}{l}53,40 \\
89,00\end{array}$ & $\begin{array}{l}53,40 \\
89,00\end{array}$ & $\begin{array}{r}71,20 \\
106,80\end{array}$ & $\begin{array}{l}53,40 \\
71,20\end{array}$ \\
\hline oxygène $z^{\text {minn }} \begin{array}{l}\max \\
\max \end{array}$ & $\begin{array}{r}82 \\
115\end{array}$ & $\begin{array}{r}85 \\
107\end{array}$ & $\begin{array}{r}80 \\
107\end{array}$ & $\begin{array}{r}85 \\
112\end{array}$ & $\begin{array}{r}75 \\
107\end{array}$ & $\begin{array}{r}88 \\
100\end{array}$ & $\begin{array}{r}84 \\
117\end{array}$ \\
\hline
\end{tabular}

Les Plécopteres adultes ont été capturés, soit à l'aide d'un filet entomologique en fauchant la végétation herbacée, arbustive et arborescente tout près de l'eau courante, soit parmi et au-dessous des galets et des pierres des grèves et des berges. Pendant le semestre froid les récoltes ont été effectuées surtout à vue sur la neige.

Les nymphes ont été prélevées à l'aide d'une passoire (vide de maille: $1 \mathrm{~mm}$ ) posée en aval des pierres immer. gées que l'on retournait. Les exuvies ont été récoltées sur les blocs, les piles des ponts et parmi les pierres des berges.

Lors des récoltes et dans toutes les stations, les principales caractéristiques physico-chimiques de l'eau ont été enregistrées. La température a été déterminée a l'aide de thermomètres à mercure (échelle de $0,5^{\circ} \mathrm{C}$ ), le $\mathrm{pH}$ au moyen de l'indicateur liquide Merk (échelle de 0,5 unité $\mathrm{pH})$; la dureté totale par le test Aquamerk. L'oxygène dissous a été dosé d'après la méthode de Winkler, en calculant les pourcentages par tapport à la saturation avec l'abaque de Mortimer (Hutchinson 1957).

Le tableau I présente les valeurs minimales et maximales des mesures effectuées pendant l'annèe entière dans. les stations au-dessous de $1.400 \mathrm{~m}$ et durant une période plus ou moins longue du printemps à l'automne dans les stations les plus élevées inaccessibles en hiver en raison de l'enneigement, du gel ou du danger d'avalanches.

La temperature des cours d'eau etudiés varie en fonction de l'al titude, ou de l'exposition géographique. Pendant l'hiver les températures des eaux du Pó, qui coule d'ouest en est, sont basses dans toutes les stations, notamment celles de haute altitude oì l'eau gèle en surface. En été les températures s'élèvent régulièrement d'amont en aval, atteignant un maximum de $19^{\circ} \mathrm{C}$ dans la station la plus basse (tableau I). L'amplitude thermique annuclle est faible dans la zone des sources du Pồ à $2.020 \mathrm{~m}$ (les températures estivales en plein jour n'excèdent pas $7^{\circ} \mathrm{C}$ ), mais elle est de plus en plus forte dans les stations d'aval. L'amplitude diume estivale at teint $5^{\circ} \mathrm{C}$ à $2.020 \mathrm{~m}$ et $6^{\circ}$ C à $1.700 \mathrm{~m}$, le réchauffement étant très important pen. dant le jour.

Les deux ruisseaux affluents présentent l'un par rapport à l'autre des caractéristiques thermiques bien différentes (Tableau I). Le Martino (station 6), qui jaillit de la grotte du même nom, coule dans un ravin de quelques centaines de mètres avant de se jeter en rive droite du Pô ; son amplitude therrnique annuel le est très faible $\left(2,5^{\circ} \mathrm{C}\right)$ et $\mathrm{I}^{\prime} \mathrm{ampli}$. hude diu rne du mois le plus chaud est inférieure à $0,5^{\circ} \mathrm{C}$. Au contraire le Tossiet prend sa source à l'altitude de 2.200 m et, avant de confluer en rive gauche du Pó (station 7), coule en direction nord-sud dans un vallon en pente raide; l'amplitude annuelle mesurée est de $11^{\circ} \mathrm{C}$ et l'amplitude diurne du mois le plus chaud de $6^{\circ} \mathrm{C}$. Il faut noter que le débit de ce ruisseau est faible, tandis que l'insolation est forte pendant les heures de la mi-journée.

Le pH est compris entre 7,0 et 7.5 dans la plupart des stations. Dans la zone des sources du Pô un pH de 6,5 a été enregistré au mois de mai au début de la fonte des nejges qui couvraient encore abondamment le Piano del Re. Dans les stations 2 et 7 les valeurs du pH mesuré ont été constamment égales à 7,0 .

La dureté totale est en général assez basse, seules les eaux du Martino sont plus minéralisées puisqu'elles filtrent à travers des roches calcaires avant la résurgence de la grotte (Tableau I).

L'action de l'homme, à peu près nulle dans les deux ruisseaux, est de plus en plus polluante dans le Pô, à mesure que l'on va de la source vers le bas de la vallée. On note déjà une faible pollution du Piano del Re, où, pendant l'été, de nombreux excursionnistes pique-niquent, lavent leur 
vaisselle avec des produits détergents et rejettent beaucoup d'ondures dans le Pô. La pollution s'accroît à Crissolo par suite du déversement des eaux ménagères et des égouts (IRSA 1977). La même chose se répète à Paesana, où s'ajoutent les effluents déversés par quelques étables et porcheries; le débit du Pô ne peut diluer ces polluants qu'à l'épor que des hautes eaux printanieres et automnales. De plus, il faut ajouter qu'au niveau de Crissolo, dans les caux du Pó, ont été enregistrées des concentrations sersibles en métaux lourds tels que $\mathrm{Ni}, \mathrm{Pb}, \mathrm{Cu}, \mathrm{Zn}$ et d'insecticides surtout DDT et dichlorophényléthane (IRSA 1977). Cette présence ne peut s'expliquer que par des retombées de pro duits transportés en amont par les vents venus de la plaine.

La dégradation de la qualité des eaux du cours de montagne du Pô est donc assez sensible et a amené la raréfaction et même la disparition des espèces les moins tolérantes à la pollution.

\section{Les espèces recensées}

Dans cette liste, les 55 espèces recensées ont été rangées d'après le système de classification phylogénétique proposé par Zwick (1973, 1980).

Le peuplement plécoptérique des biotopes lotiques étudiés est en règle générale typique des cours d'eau des Alpes occidentales (Aubert 1986, Consiglio 1976, Rauser 1971). Les 7 familles connues de Plécoptères européens y sont représentées.

\section{Perlodidae}

1 Dictyogenus alpinum (Pictet) 1842

2 Perlodes intricatus (Pictet) 1842

3 microcephalus (Pictet) 1842

4 Isoperla carbonaria Aubert 1953

5 grammatica (Poda) 1761

6 oxylepis (Despax) 1936

7 rivulorum (Pictet) 1842

\section{Perlidae}

8 Dinocras cephalotes (Curtis) 1827

9 Perla grandis Rambur 1841

10 marginata (Panzer) 1799

\section{Chloroperlidae}

11 Chloroperla susemicheli Zwick 1967

12 Siphonoperla montana (Pictet) 1842

13 torrentium (Pictet) 1842

\section{Taeniopterygidae}

14 Rhabdiopteryx alpina Kühtreiber 1934

15 Taeniopteryx kühtreiberi Aubert 1950

\section{Nemouridae}

16 Amphinemura sulcicollis (Stephens) 1836

17 Protonemura ausonia (Consiglio) 1955

18 brevistyla $\mathrm{R}$ is 1902

19 caprai Aubert 1954

20 intricata Ris 1902

21 lateralis (Pictet) senst Ris 1902

22 nimborella Mosely 1930

23 nimborum Ris 1902

24 nitida (Pictet) sensu $\mathrm{R}$ is 1902

25 praecox (Morton) 1894

26 Nemoura cinerea selene Consiglio 1959

27 mortoni Ris 1902

28 oropensis Rav. \& Rav. Dem. 1980

29 palliventris Aubert 1953

30 sinuata $\mathrm{R}$ is 1902

31 uncinata Despax 1934

(fulviceps Klapalek 1902 sensu

Aubert 1956 nec Klap.)

32 Nemurella pictetii Klapalek 1909

\section{Capniidae}

33 Capnia bifrons (Newman) 1838

34 nigra (Pictet) 1942

35 vidua vidua Klapalek 1904

\section{Leuctridae}

36 Lenctra alpina Kühtreiber 1934

37 armata Kempny 1899

38 elisabethae Ravizza 1985

39 fusca (Linné) 1758

40 handlirschi Kempny 1898

41 helvetica Aubert 1956.

42 hippopus Kempny 1898

43 inermis Kempny 1899

44 leptogaster Aubert 1949

45 major Brinck 1949

46 meridionalis Aubert 1951

47 moselyi Morton 1929

48 nigra Olivier 1811

49 niveola Schmid 1947

50 rauscheri Aubert 1957

51 rosinae Kempny 1900

52 schmidi Aubert 1946

53 sesvenna Aubert 1953

54 teriolensis Kempny 1900

55 vesulensis Rav. \& Rav. Dem. 1984 


\section{Les stations et leur faune}

Les données relatives à chaque station sont réparties dans deux rubriques : description de l'environnement et espèces récoltées.

Dans la liste des espèces nous nous bornons à donner le total des spécimens recueillis d'après leur fréquence décroissante, en utilisant les abréviations suivantes: $\sigma \circ / q$ \% $=$ nombre de spécimens adultes mâles et femelles; ny = nymphes $: \mathrm{ex}=$ exuvies : chiffres romains $=$ premier et dernier mois de la période de vol. Abréviations des genres : $A .=A m p h i+$ nemura; $\mathrm{Ca}=$ Capnia $\mathrm{Ch}=$ Chloroperla $; \mathrm{Dc}$. Dictyogenus; Dn. = Dinocras; $I$. Isoperla ; L. Leuctra; $N .=$ Nemoura $; N l .=$ Nemurella $; P .=$ Protonermura; Pe. $=$ Perla $; P l=$ Perlodes $\boldsymbol{R} .=R h a b$ diopteryx $;.=$ Siphonoperla $T .=$ Taeniopteryx.

5.1. Station 1, la zone des sources du Pó, au Piano del Ke, $2.020 \mathrm{~m}$.

Le Pô jaillit brusquement d'un amas de roches éboulées et est alimenté sur ses 100 premiers mètres de cours par quelques sources secondaires et deux ruisselets, puis il coule en pente douce à travers une prairie bourbeuse et dépourvue de végétation arbustive. Son lit, large de 2 à $5 \mathrm{~m}$, où en débit moyen l'eau atteint une profondeur de $30 \mathrm{~cm}$, est garni de gros blocs et de pierres recouvertes en partie de mousses. A proximité de l'eau se trouvent de nombreux Caltha palustris, Epilobium alpinum, Saxifraga stellaris, Carex flava et Cardamine amara (IRSA 1977). L'enneigement et le gel se prolongent de fin octobre à inai.

Quinze séries de récoltes, de mai à octobre, ont permis de capturer 20 espèces.

P. brevistyla 270/409, 2 ny, 6 ex (VI-X); P. nimborella $110 / 65$ (IX-X); L. rauscheri $61 / 109$ (VI-VIII) ; P. nimborum 29/55, 4 ny, 65 ex (V-VII) ; P. lateralis 72/43, 28 ny. 11 ex (VI-IX); L. armata 35/43 (VI-VII) ; I. rivulorum 33/24, 6 ny (VI-X); Pl. intricatus 7/18, 1 ny, 64 ex (V-IX); S. montana 9/18, 1 ex (VI-VIII); Dc. alpinum 1/9, 2 ny, 16 ex (VI-VIII); L. rosinae 13/10 (VIIX); L. teriolensis $14 / 8$ (VI-VII) ; $N$. siruata $6 / 4,11$ ny (VI-VII); $P$. nitida $3 / 8(\mathrm{IX}-\mathrm{X}) ; L$. helvetica $3 /-(\mathrm{V})$; A. sulcicollis -12 (VII) $;$. aropensis $1 /$ (VI) $; N$. mortont $1 /$ - (VI) ; L. alpina 1/- (VI) ; L. sesvenna -11 (VI).

\subsection{Station 2, le Pô au Piano della Regina, $1.700 \mathrm{~m}$.}

Dans cette station, située près de $3 \mathrm{~km}$ en aval de la précédente, le $P \hat{o}$, qui a reçu les eaux de plusieurs ruisselets, coule en pente douce à travers une prairie alpine dans un lit large de 5 a $8 \mathrm{~m}$, sur un fond de pierres, de cailloutis et de graviers. En débit moyen la profondeur de l'eau est de $30 \mathrm{~cm}$. Des mousses abondent sur les pierres des berges mouillées par le courant ; sur les rives se trouvent de rares arbustes: Salix et Ainus. L'enneigement se prolonge de novembre à avril-mai.

Dix-neuf séries de prélèvements, de mars à novembre, ont permis de récolter 22 espèces.

L. rauscheri $237 / 340$ (V-VIII); P. nimborum 227/151, 50 ny (V-VII) ; N. mortoni 108/134, 1 ny, 4 ex (V-VIII) ; $P$. nitida 86/73, 2 ny, 18 ex (IX-XI) ; $P$. lateralis 50/48, 13 ny (V-XI) ; I. rivulorum 24/27, 14 ny, 4 ex (V-VIII) ; P. brevistyla 21/23, 8 ny (VIII-IX); Ca. vidua vidua $24 / 16$ (III-IV); $D c$, alpinum $3 / 2,8$ ny, 12 ex (VI-VII) ; Pl. intricatus 1i-, 4 ny, 8 ex (VI-VII) ; S. montana 3/8, 1 ny (VI-VIII); $R$. alpina 5/-, 2 ex (V); L. helvetica 3/4 (IV-VI); L. alpina 4/1 (V-VI); P. nimborella $1 / 1,1$ ny $(\mathrm{X})$; L. inermis $-/ 2$ (VI) ; L. teriolensis $1 / 1$ (VIII); L. schmidi $t 2$ (IX); 1. carbonaria $1 /$ (VI) ; Ch. susemicheli $/ 1$ (IX); Ca. nigra t1 (VI) ; $L$. sesvenna $/ 1(\mathrm{~V})$.

\subsection{Station 3, le Pô à Crissolo, $1.380 \mathrm{~m}$.}

Peu en amont de Crissolo, le Pô coule à travers une hêtraie dans un lit large d'une douzaine de mètres et garni de gros blocs erratiques, de pierres et de graviers. Les pierres de la base des berges abruptes sont abondamment recouvertes de mousses. Les deux rives portent une abondante végétation herbacée où prédominent Saxilraga aizoides, Festuca rubra et Carex frigida, et arbustive avec de nombreux Salix et Alnus. La durée d'enneigement varie suivant les années et s'étend en moyenne de novembre à mars.

Vingt-huit séries de récoltes réparties sur tous les mois de l'année ont permis de capturer 31 espèces. Aucun adulte n'a été trouvé de janvier à la mifevrier,

P. nitida 74/212, 24 ny (IX-XII) ; P. nimborum 65/21, 112 ny (IV-Vi); L. helvetica 84/64, 3 ny (III-V); Ca. vidua vidua $78 / 45$ (II-IV); L. rauscheri $35 / 57$ (V-IX); I. rivulortm 20/20, 16 ny (V-VIII) : L. inermis 14/28 (V-VII) ; L. moselyi 17/24 (VIII-XI) ; N. mortoni 4/5, 
20 ny (V) : Ch. susemicheli $4 / 33,3$ ex (VIII-XI); $L$. schmidi 13/24 (VIII-XII) ; P. lateralis 7/1 1 (IV, VI-IX) ; Pl. intricatus 11 ny, 2 ex (VI-VII) ; R. alpina 6/2,3 ny (IV); L. niveola 5/6 (III-IV); L. alpina 4/3, 2 ny (V.VI); A. sulcicollis $1 / 6$ (VII) : P. brevistyla $-/ 1,4$ ny (VIII); N. sinuata 15 (VII, X); L. major 15 (X-XI) ; I. carbonaria 1/3 (VI-VII): Dc. alpinum $1 /-, 1$ ny, 1 ex (V); $L$. teriolensis -13 (VIII); L. neridionalis $-12(\mathrm{X}-\mathrm{XI}) ; L$. vesulensis $1 / 1(\mathrm{X}) ;$ Pl. microcephalus $1 /-(\mathrm{V}) ; \mathrm{S}$. montana -11 (VII) ; S. torrentium $/ \mathrm{I}$ (VII) ; L. leptogaster $1 /$ - (XI) ; P. nimborella $/ 1$ (X); T. kühtreiberi 1 ex (TV).

\subsection{Station 4, le Pô peu en amont de Paesana, vers 650 m.}

Le Pô creuse son cours dans ses propres alluvions. Le lit, large de plus de $25 \mathrm{~m}$, est composé de gros blocs, de pierres de dimensions très variables et de graviers. Un barrage situé quelques centaines de mètres en amont entraîne un détournement partiel des eaux réduisant au tiers du lit le cours vif lors des débits moyens. Les maigres débits estivaux et hivernaux sont donc plus marqués que dans les stations situées en amont. Les berges très escarpées sont surplombées de feuillus divers et de prés de fauche. L'enneigement, peu abondant, est limité aux mois d'hiver.

Vingt et une séries de récoltes réparties sur tous les mois de l'année ont permis de recenser 26 espèces. Pas d'adultes en janvier.

L. elisabethae 121/113, 3 ny (II-V); A. sulcicoltis 43/142, 1 ny (VI-VII) ; L. hippopus $92 / 70$ (IV-VI) ; $L$. meridionalis 43/49 (VIII-XII); N. uncinata 8/12, 59 ny (IV-V); L. niveola 30/7, 1 ny (II-IV): I. carbonaria 5/9, 11 ny (V-VI) ; $P$. nitida $2 / 27$ (X-XII) ; Dn. cephalotes 4/1. 14 ny. 9 ex (VI) ; L. fusca 4/13 (X-XII); P. intricata 3/13 (VI-VII); L. alpina 4/7 (IV); P. praecox $4 / 4,1$ ny (III) ; L. leptogaster $4 / 5$ (X-XII) ; S. torrentitum 3/5 (VI); I. grammatica 2/-, 4 ny (VI); L. rauscheri $1 / 3$ (V-VII); Pl. microcephalus 1/-, 1 ny, 1 ex (V); Pe. marginata 3 ny ; Ca. vidua vidua $2 / 1$ (III) : N. cine . rea selene 1/- (VI) ; N. palliventris $1 /$ - (IV) ; L. handlirschi -11 (VI) ; L. helvetica 1/- (II) ; L. moselyi $/ 1$ (VIII).

\subsection{Station 5, le Pô entre Paesana et Sanfront, vers $550 \mathrm{~m}$.}

Située $3 \mathrm{~km}$ en aval de la précédente, cet te station en a les mémes caractéristiques en ce qui concerne la largeur du lit, le débit et la végétation des berges.
Cependant le fond est vaseux et les eaux sont polluces par des effluents d'étables et de porcheries. La pollution est plus marquée pendant les étiages d'été et d'hiver ; la vase du fond est alors imprégnée de lisier, surtout le long des rives où le courant est le plus faible.

Quatorze séries de récoltes réparties sur tous les mois de l'année ont permis de découvrir 15 espèces. Pas d'adultes de décembre à février et de la mi-juin à septembre.

L. hippopus 58/51, 3 ny (III-IV); N. uncinata 21/14, 59 ny (III-V); L. elisabethae 27/27, 2 ny (III-IV); $P$. intricata 22/22, 10 ny (V); A. sulcicollis $28 / 20$ (V-VI) ; N. cinerea selene $13 / 9, \mathrm{I}$ ny $(\mathrm{V})$; Ca. bifrons $10 / 5$ (IIIIV); L. fusca $3 / 4$ (X-XI) ; L. nigra $3 / 1$ (V); I. grammatica $1 / 2(\mathrm{~V}) ;$ L. inermis -13 (IV-V); l. oxylepis $2 /$ - (V); I. carbonaria $1 / 1$ (V); P. praecox $2 /$ - (III) ; P. nitida -1 (XI).

\subsection{Station 6, le ruisseau Martino à Crissolo, 1.380-1.400} m.

Ce ruisseau jaillit de la grotte du " rio Martino" vers $1.450 \mathrm{~m}$ et se jette dans le Pồ à $1.380 \mathrm{~m}$ (rive droite). L'eau dévale, en une succession de rapides, dans un ravin très ombragé par suite de la végétation ou de l'exposition nord et de la forte pente. Le lit, large de 2 à $3 \mathrm{~m}$, est constitué de blocs et de pierres recouvertes de mousses. Le ruisseau est situé dans une hêtraie-sapinière; parmi la végétation hygrophile des rives prédominent Salix, Alnus et Petasites officinalis. Période d'enneigement comme dans la station voisine $\left(\mathrm{n}^{\circ} 3\right)$.

Vingt-huit séries de récoltes réparties sur tous les mois de l'année ont permis de recenser 27 espèces. Pas d'adultes en janvier et février.

L. helvetica 246/176 (III-IV); P. lateralis 82/108, 35 ny (V-XI) ; L. vesulensis 95/84, 12 ny (IX-XI) ; L. rauscheri $68 / 113(\mathrm{~V}-\mathrm{X})$; P. nimborum 83/37, 12 ny, $2 \mathrm{ex}$ (IV-VI) ; L. schmidi 56/38, 8 ny (VIII-XI) ; P. nittida $27 / 55,23$ ny (IX-XII) ; I. rivulorum 32/31, 20 ny (V. X) ; L. niveola 25/41 (III-IV); Ca. vidua vidua $17 / 23$ (III-IV); $L$. inermis $22 / 18$ (V-VII) ; L. teriolensis $9 / 27$ (VIII-X); L. moselyi $10 / 18,1$ ny (VIII-XI) ; N. sinuata $8 / 9,9$ ny (V-IX); $N$. mortoni $7 / 9,1$ ny (V-VII); $L$. armata $4 / 13$ (V-VII) ; P. caprai $5 / 5,6$ ny (V, VII-IX); Ch. susemicheli t9 (VIII-X); L. alpina $1 / 5$ (V-VI); L. major 1/5 (IX-X); $P$. ausonia 3/1, 1 ny (IX-XII) ; Pl. intricatus $/ 1(\mathrm{~V})$; Pl. microcephalus $/ 1$ (VI) ; A. sulcicollis $/ 1$ (VII) : P. brevistyla $/ 1$ (VI) ; P. nimborella $f 1(\mathrm{X}) ; L$. meridionalts $1 / \mathrm{X}(\mathrm{X})$. 
5.7. Station 7, le Tossiet en aval de Crissolo, 1.150$1.200 \mathrm{~m}$.

Avant de déboucher dans le Pó à environ $1.130 \mathrm{~m}$ (rive gauche), le Tossiet coule dans un vallon escarpé, exposé au sud (adret). Du fait de la présence de nombreux blocs éboulés, plusieurs petites cas. cades se succèdent le long de son cours. Dans les secteurs à pente moins forte, le lit, d'une largeur très variable, est constitué de pierres, de graviers et de sable. De nombreux $A$ lnas el Salix, bordent le ruisseau qui traverse un bois de hêtres et autres feuillus.

Vingt-quatre séries de récoltes réparties sur tous les mois de l'année ont permis de capturer 30 espèces. Pas d'adultes de la mi-ćécembre à la mi-janvier. L. inemis $154 / 117,31$ ny (IV-VI) ; L. alpina 141/69, 19 ny (IV-V); L. niveola 123/95, 1 ny (I-V); L. helvetica $70 / 58$ (II.V); P. nimboram 65/39, 8 ny (III-IV) : I. carbonaria 19/12, 4 ny, 35 ex (V-VII); A. sulcicollis $42 / 26$ (V-VIII) ; $N$. mortoni 18/10, 4 ny (IV-V) ; $L$. elisabethae $12 / 18$ (I-IV): N. uncinata 13/5. 7 ny (IV. V) ; $P$. intricata 4/15 (VI-VII) ; $P$, nitida $3 / 12$ (X-XII); Pe. grandis $-11,14$ ex (VI-VII) ; $N$. sinuata 5/9 (V-VIII) ; P. caprai $7 / 4$ (VI-VII); $P$. lateralis 37,1 ny (VI-VII) ; L. major 3/6 (IX-XI); L. moselyi 5/3 (VIII-X); $P$ l. microcephalus $1 / 1,3$ ny, 2 ex (IV-V); Dc. alpinum 6 ex (V.VI) ; L. rauscheri $5 / 1$ (IV-V); L. armata $5 /$ - (V); L. meridionalis $2 / 3$ (VII-X); S. correntium 2/1 N.VI); R. alpina $2 / 1$ (IV) : L. hippopus $/ 3$ (V-VI); L. leprogaster $2 / 1$ (IX-X); Dn. cephalotes 1 ex (VI); Nl. picte tii 1/-(VI) ; L. schmidi -l/ (X).

\section{Répartition altitudinale}

Plusieurs auteurs ayant étudié l'écologie des Plécoptères d'eau courante des régions montagneuses d'Europe (Berthélemy 1966, Brinck 1949, Kamler 1967, Lillehammer 1974), ont mis en évidence le rôle principal joué par la température dans la distribution spatiale des espèces. La répartition altitudinale des Plécoptères dans la valléc du Pô (Tableau II) et la distribution relative des espèces, sont conformes aux données résultant de l'étude d'autres vallées des Alpes occidentales (Gay 1982, Ravizza \& Ravizza Dematteis 1976, 1986, Ravizza Dematteis \& Ravizza 1983a).

Dans les stations du cours supérieur, la plupart des espèces sont orophiles et sténothermes d'eau froide. A l'état nymphal, elles trouvent dans les cours d'eau d'altitude leurs conditions optimales de développement. Parmi ces espèces, certaines, strictement alticoles, sont des composantes typiques de la faune de Plécoptères des cours d'eau de l'étage subalpin (altitudes en moyenne supérieures à 1.900 m), d'autres, plus nombreuses, ont une répartition extensive dans l'étage montagnard. Au premier ensemble appartiennent Protonemura brevistyla, $P$. nimborella, Nemoura oropensis, Leuctra rosinae et L. sesvenna; au deuxième, Perlodes intricalus, Dictyogenus alpinum, Isoperla rivilontm, Chloroperla susemicheli, Siphonoperla montana, Rhabdiopteryx alpina, Taeniopteryx kühtreiberi, Protonemura lateralis, $P$. nimborum, Nemoura mortoni, $N$. sinuata, Capnia vidua vidua, Leuctra armata, $L$. helverica, $L$. moselyi, $L$, rauscheri et $L$. ieriolensis.

C'est dans les cours d'eau entre 1.000 et $1.400 \mathrm{~m}$, que la diversité de la faune de Plécoptères est maximale : en effet, aux espèces orophiles du deuxième ensemble s'ajou tent celles qui se développent principalement dans les ruisseaux montagnards, à savoir Isoperla carbonaria, Siphonoperla torrentium, Profonemura ausonia, $P$. caprai, Leuctra niveola, $L$. schmidi et L. vesulensis, ou celles qui ont une large valence écologique, telles que Perlodes microcephaIus, Perla grandis, Amphinemura sulcicollis, Protonemura nitida, Nemurella pictetii, Capnia nigra, Leuctra alpina, L. inermis, L. leptogaster et L. major.

Dans les stations au-dessous de $700 \mathrm{~m}$, la diversité et la richesse de la faune de Plécoptères décroissent par suite de la disparition progressive des formes orophiles, remplacées par un nombre plus faible d'espèces eurythermes de basse altitude, et par suite de l'augmentation de la pollution. Parmi les espèces qui habitent surtout le cours submontagnard du Pô, il faut noter Perla marginata, Dinocras ceplatotes, Isoperla grammatica, I. oxylepis, Amphinemura sulcicoltis, Protonemura intricata, $P$. praecox, Nemoura cinerea selene, $N$. palliventris, $N$. uncinata, Capnia bifrons, Letcira elisabechae, L. fusca, $L$. handlirschi, L. hippopus, L. inemis, L. meridionalis et $L$. nigra. Dans la station la plus basse, $550 \mathrm{~m}$, survivent les espèces les plus tolérantes vis-à-vis de la pollution, parmi lesquelles Nemoura uncinata et Leuctra hippopus sont dominantes et Amphinemura sulcicollis, Protonemura intricata, Nemoura cinerea selene et Leuctra elisabethae sont assez abondantes.

Berthélemy (1966) avait montré que dans certaines hautes montagnes d'Eu rope telles que les Alpes et les Pyrénées, les espèces de Plécoptères d'un 
Tableau II. Répartition en altitude des Plécoptères de la vallée supérieure du Pó

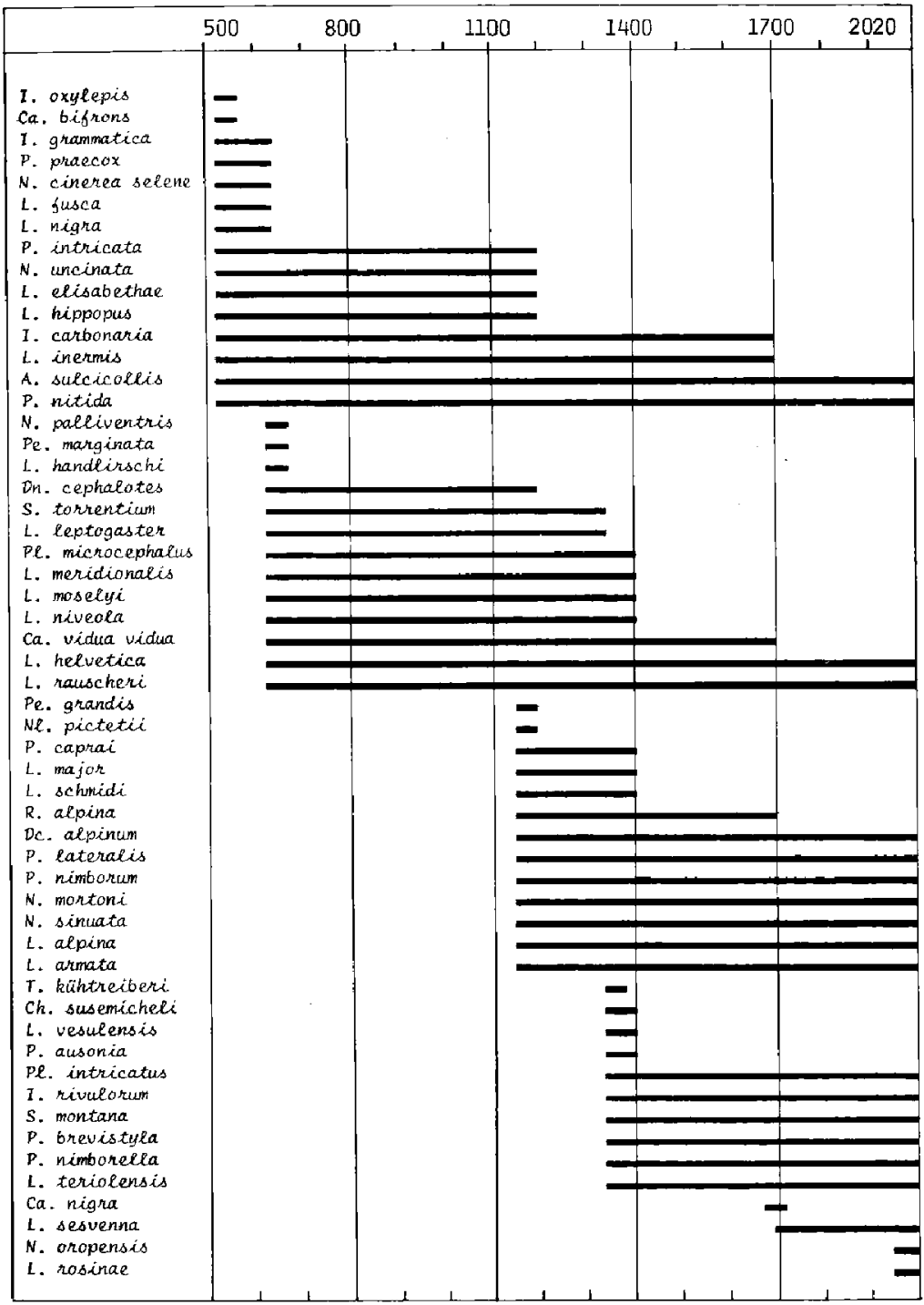


mème genre se remplacent régulièrement d'amont en aval. Cette succession altitudinale a aussi été vérifiée dans la vallè du Pô. C'est le cas, par exemple, des formes orophiles Perlodes intricatus, Isoperla rivulorum, Siphonoperla moniana, Protonemura nimbortum, Nemoura mortoni, Capnia vidua vidua, Leuctra rosinae et $L$. rauscheri remplacées en aval respectivement par Perlodes microcephalus, Isoperla grammatica, Siphonoperla torrentium, Protonemura praecox, Nemoura uncinata, Capnia bifrons, Leuctra hivpopes et $L$. inermis.

\section{Périodes de vol}

Dans les cours d'eau des Alpes, la courbe des émergences des Plécoptères adultes est unimodale ou bimodale, suivant l'altitude de la station.

Ainsi, dans l'étage submontagnard de la vallée du Pô, entre 400 et $1.000 \mathrm{~m}$, on observe deux périodes de vol distinctes, l'une de la fin de l'hiver au début de l'été, l'autre de la fin de l'été à l'automne tardif (Tableau III). L'interruption des émergences (hivernale ou estivale) est liée surtout à la température de l'eau et secondairement à la photopériode (Hynes 1976); l'émergence des adultes est inhibée lorsque l'eau est trop chaude et la durée du jour trop longue (été), ou bien lorsque l'eau est trop froide et la durée du jour trop courte (hiver).

Avec l'altitude, l'interruption estivale diminue progressivement; elle disparaít à partir de l'étage montagnard, au-dessus de 1.000-1.200 m, où il n'y a plus qu'une seule période de vol qui s'étale du printemps à l'automne. Par contre, l'interruption pendant la saison froide est d'autant plus longue que l'altitude augmente.

Plécoptères hivernaux. Les mues imaginales ont lieu durant les premiers mois de l'année. Dans le Tossiet, grâce à une exposition en adret (sud), les premiers adultes de Letictra elisaberhae et $L$. niveola apparaissent pendant la seconde quinzaine de janvier, suivis en février par ceux de Leuctra helvetica et en mars, de Protonemura nimbortum. Le début des mues imaginales de ces mèmes espèces est en moyenne retardé de 2 à 4 semaines dans les autres stations plus froides par suite de leur exposition moins favorable, ce qui entraine le phénomène de l'inversion des températures. Parmi les espèces qui éclosent vers la fin de l'hiver il faut noter encore Protonemura praecox et Capnia bifrons, cantonnées en basse altitude, et Taenioptervx kühtreiberi et Capmia vidua vidua, qui habitent les cours d'eau de l'étage montagnard. La période de vol de la plupart des formes hivernales s'etale plus ou moins tard durant le printemps.

Plécoptères printaniers. En dessous de $1.300 \mathrm{~m}$, les premiers adultes apparaissent courant avril, c'est le cas de Rhabdioptervx alpina, Protonemura intricata, Amphinemura sulcicollis, Nemoura cinerea selene, $N$. palliventris, $N$. uncinata, Leuctra alpina, L. hippoptis, $L$. inermis et $L$. nigra, puis à partir de mai à juin apparaissent Perlodes microcephalus, Dictyogenus alpinum, Isoperla carbonaria, $I$. grammatica, I. oxylepis, Dinocras cephalotes, Perla grandis, Siphonoperla torrentium, Protonemura lateralis, Nemoura mortoni, $N$. sinuata, Nemurella pictetii, Leuctra armata, $L$. handlirschi et $L$ rauscheri. Au-dessus de $1.300 \mathrm{~m}$ les émergences des espèces hivernales se prolongent, jusqu'au mois d'avril qui, à cette altitude, correspond encore à l'hiver. Les adultes des formes printanières n'apparaissent que courant mai ; ce sont des espèces à large valence écologique énumérées ci-dessus, ou des formes sténothermes d'eau froide répandues jusque dans l'étage subalpin, telles que Perlodes intricatus, Isoperla rivulorum et Leuctra teriolensis, auxquelles s'ajoutent, de mai à juin, Nemoura oropensis et Leuctra sesvenna cantonnées seulement en altitude.

Plécoptères orophiles estivaux. Dans les étages montagnard superieur et subalpin, aux especes hivernales et printanières habituellement peu nombreuses en altitude, succèdent sans discontinuité, les espèces estivales dont les émergences s'étalent, suivant l'altitude, de mai-juin jusqu'en aoûtseptembre. Ce sont Perlodes intricatus, Isoperla rivulorum et Letucira teriolensis qui ont une longue période de vol s'étendant sur 4 ou 5 mois et Siphomoperla montana, Chloroperla susemicheli, Protonemura brevistyla et Leuctra rosinae.

Plécoptèrers automnaux. Dans l'étage montagnard, les premiers adultes apparaissent vers la fin août ; c'est le cas de Leuctra moselyi et $L$. schmidi; et c'est entre septembre et octobre qu'émergent les autres formes d'automne Protonemura nimborella, $P$. nitida, Leuctra fusca, L. leptogaster, L. major, L. meridionalis et $L$. vesulensis. A l'exception de P. nimborella, cantonnée en haute altitude, qui vole seulement de septembre au début de novembre, les autres espèces ont une période d'activité imaginale plus 
Tableau III. Périodes de vol des Plécoptères dans la vallée supérieure du Pô : A dans la station 1, à $2.020 \mathrm{~m}$; B dans les stations 3 et 6 , vers $1.380 \mathrm{~m} ; C$ dans la station 5 , à $650 \mathrm{~m}$.

A

P. nimborum

L. rauscheri

Pe. intricatus

L. armata

Oc. alpirtum

N. sinuata

[. teriolensis

S. montana

L. rosinue

P. Rateralis

I. hivularam

P. brevistyla

P. nimborella

P. nitida

\section{$\mathbf{B}$}

Ca. vidua vidua

1. niveora

L. helvetica

P. nimborum

R. alpina

L. alpina

P. Lateralis

DC. alpivtum

N. mortoni

L. armata

L. inermis

N. sintcata

L. rauscheri

Pl. intricatus

P. caprá

I. rivularum

L. teriolensis

ch. susemicheli

L. maselui

1. schmidi

1. majar

1. vesulensis

P. ausonia

p. nitida

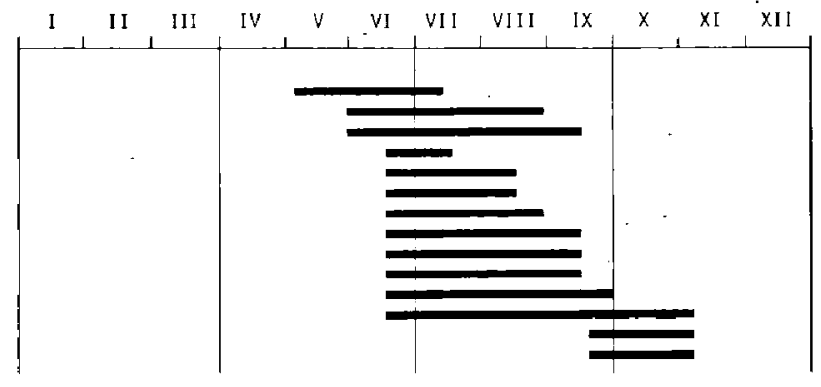

\section{c}

1. elisabethre

1. niveola

P. pha ecox

N. uncinata.

L. inermis

1. hippopus

1. alpina

De. microcephalus

I. carboraria

L. rauscheri

Dr. cephalotes

A. sulcicollis

P. intricata

S. torsentium

l. meridionalis

L. leptogaster

P. nitida

L. fusca

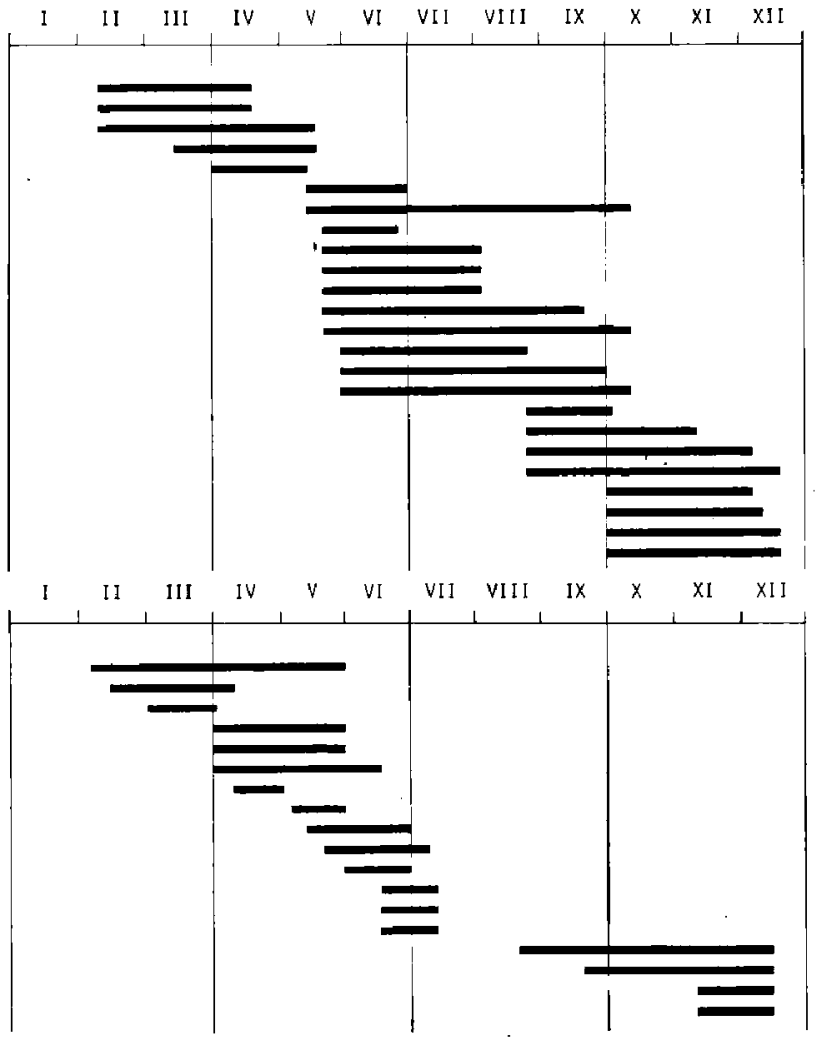


longue qui s'étale pendant une grande partie de l'au tomne.

Les tableaux III A, B, C montrent l'influence de I'altitude sur la période de vol dans la vallée du Pô. A quelques exceptions près, les espèces rares et dispersées n'y sont pas incluses.

\section{Notes biogéographiques}

La plupart des Plécoptères de la vallée du Pô sont répandus danś les Alpes du Piémont et aussi dans les Alpes françaises (Aubert 1986), à l'exception de quelques espèces endémiques de la région italienne.

Environ $71 \%$ des espèces recensées ont une répartition européenne plus ou moins extensive, tandis que les $29 \%$ restant sont des formes alpines et apennines.

Dans ce paragraphe nous mentionnons seulement les espèces dont la présence dans cette vallée est intéressante du point de vue biogéographique.

L'espèce la plus remarquable est sans doute Leuctra vesulensis, sténoendémique de la vallée du Pô (Ravizza \& Ravizza Dematteis 1984). A l'exception de deux mâles récoltés sur la rive gauche du Pô (station $3)$, nous n'avons trouvé cette espèce que dans le Martino (station 6). Il s'agit d'une forme à ailes réduites dans les deux sexes, apparentée à une autre espèce brachyptère, Leuctra brevipentiis Rav. (Ravizza 1978, Ravizza \& Ravizza Dematteis 1982), sténoendémique des mont du Biellais. On peut vraisemblablement considérer ces deux espèces automnales, qui par leurs caractères morphologiques sont séparées d'une manière nette des congénères du groupe hippopus (sensu lato), comme des formes relictes préglaciaires. D'après Jeannel (1942), les monts du Biellais (Alpes Pennines) et le mont Viso (Alpes Cottiennes), grâce à leur position avancée sur la bordure interne des Alpes occidentales, ont été des * massifs d'attraction " qui ont récupéré après les périodes glaciaires leurs propres espèces et celles des massifs voisins, chassées par l'extension des glaciers. Cette hypothèse basée surtout sur l'étude des Coléoptères Carabiques Trechinae, s'applique également bien aux cas de Leuctra vesulensis et $L$. brevipennis. Le brachyptérisme qui caractérise ces Leuctra laisse supposer que leurs anciennes aires de répartition étaient peu étendues et que ces espèces étaient déjà cantonnées dans des biotopes lotiques isolés.
Deux especes strictement orophiles, Nemoura oropensis endémique des monts du Biellais (Ravizza \& Ravizza Dematteis 1980), et Leuctra sesvenna, forme alpine d'altitude répandue de manière discontinue dans les Alpes cent rales et occidentales (Aubert 1953, 1959, Ravizza Dematteis \& Ravizza 1985), n'avaient pas encore été trouvées daris les Alpes Cottiennes qui constituent leur nouvelle limite méridionale de répartition.

Isoperla oxylepis, dont la détermination nous a été confirmée par M. Berthélemy, n'avait jamais été signalée sur le versant sud des Alpes; elle est donc nouvelle pour l'Italie (zone 3 de la Limnofauna Europaea). D'après Il lies (1978), cette espèce à répartition médioeuropéenne serait "transgressante " dans la bordure septentrionale des Alpes. Dans la vallée du Pô, $L$ oxylepsis est en tous cas très rare; nous n'avons récolté que deux mâles à la station 5 .

Trois espèces apennines ont été récoltées dans la vallée étudiée, ce sont Nemoura palliventris, Protonemura caprai et $P$. ausonia. Les deux premières avaient déjà été signalées çà et là dans les Alpes du Piémont (Aubert 1954, Consiglio 1967 - sous le nom de P. costai serzsu suo nec Aubert -, Ravizza \& Ravizza Dematteis 1986); tandis que $P$. ausonia est nouvelle pour les Alpes car les localités les plus septentrionales de son aire de répartition sont Savona et Montenotte dans l'Apennin ligure (Ravizza \& Ravizza Dematteis 1983).

\section{Analyse du peuplement}

En vue d'analyser les communautés de Plécoptères de la vallée, il nous semble utile de prendre en considération les caractères se rapportant à la composition qualitative et quantitative, ainsi que la dépendance cénotique des espèces dans les 7 stations.

Nos données semi-quantitatives permettent de calculer l'abondance relative des espèces récoltées, qui, d'après Berthélemy (1966), représente " la meilleure estimation possible de leur abondance relative réelle, ou tout au moins de leurs chances d'être capturées' 3. Le tableau IV montre la répartition et l'abondance relative des espèces; pour chaque station nous avons distingué les 5 classes d'abondance suivantes:

absente

+ très rare, 1 ou 2 spécimens 
Tableau IV. Répartition et abondance des espèces dans les 7 stations.

\begin{tabular}{|c|c|c|c|c|c|c|c|}
\hline stations $n^{\circ}$ & 1 & 2 & 3 & 4 & 5 & 6 & 7 \\
\hline Dictyogenus alpinum & ++ & +++ & ++ & - & - & - & ++ \\
\hline Perlodes intricatus & $++t+$ & $+*$ & $+t$ & - & - & + & - \\
\hline microcephalus & - & - & + & ++ & - & + & ++ \\
\hline Isopenta carbonaria & - & + & ++ & +++ & + & - & ++++ \\
\hline grammatica & - & - & - & ++ & ++ & - & - \\
\hline oxylepis & - & - & - & - & + & - & - \\
\hline rivalorwm & ++ & +++ & ++ & - & - & $+++t$ & - \\
\hline Dinochas cephalotes & - & - & - & +++ & - & - & + \\
\hline Perla grandis & - & - & - & - & - & - & +++ \\
\hline marginata & - & - & - & ++ & - & - & - \\
\hline Chloroperta susemicheli & - & + & ++ & - & - & ++ & - \\
\hline Siphonoperea mantana & +++ & ++ & + & - & - & - & - \\
\hline torrentiam & - & - & + & ++ & - & - & ++ \\
\hline Rhabdiopteryx alpina & - & ++ & ++ & - & - & - & ++ \\
\hline Taeniopteryx kühtreiberi & - & - & + & - & - & - & - \\
\hline Amphinemura sulcicoleis & + & - & ++ & ++++ & ++++ & + & +++ \\
\hline Protonemura ausanis & - & - & - & - & - & ++ & - \\
\hline brevistyla & +++++ & $++*$ & ++ & - & - & + & - \\
\hline caprai & - & - & - & - & - & ++ & ++ \\
\hline intricata & - & - & - & +++ & +++ & - & +++ \\
\hline Lateralio & $++t+$ & ++++ & $+t$ & - & - & $+t+t$ & ++ \\
\hline nimbonella & +++ & ++ & + & - & - & + & - \\
\hline nimborwm & +++ & ++++4 & +++ & - & - & ++++ & ++++ \\
\hline nitida & ++ & +++ & +++++ & +++ & + & +++4 & +++ \\
\hline praecox & - & - & - & ++ & + & - & - \\
\hline Nemoura cinerea selene & - & - & - & + & +++ & - & . - \\
\hline mortoni & + & +++ & +++ & - & - & +++ & +++ \\
\hline oropens is & + & - & - & - & - & - & - \\
\hline pariventris & - & - & - & + & - & - & - \\
\hline sinuata & ++ & - & $+t$ & - & - & +++ & ++ \\
\hline uncinata & - & - & - & +++ & +++ & - & +++ \\
\hline Nemurella pictetii & - & - & - & - & - & - & + \\
\hline Capnia befrons & - & - & - & - & $+t+$ & - & - \\
\hline nigna & - & + & - & - & - & - & - \\
\hline vidua vidua & - & $+t+$ & ++++ & ++ & - & $+f$ & - \\
\hline Leuctra alpina & + & ++ & ++ & ++ & - & ++ & +++ \\
\hline armata & +++ & - & - & - & - & ++ & $4+$ \\
\hline elisabethae & - & - & - & $+t++t$ & +++ & - & +++ \\
\hline fusca & - & - & - & +++ & ++ & - & - \\
\hline handlirschi & - & - & - & + & - & - & - \\
\hline helvetica & ++ & ++ & ++++ & + & $=$ & ++++ & ++++ \\
\hline hippopus & - & - & - & ++++ & +++ & - & ++ \\
\hline inermis & - & + & $+t+$ & $t+t+$ & ++ & $t+t$ & $t+t+t$ \\
\hline leptogaster & - & - & ++ & ++ & - & - & + \\
\hline major & - & - & ++ & - & - & ++ & t+ \\
\hline meridionalis & - & - & + & +++ & - & + & ++ \\
\hline moselyi. & - & - & $+t$ & + & - & t+ & tat \\
\hline nigna & - & - & - & - & ++ & - & - \\
\hline niveala & - & - & ++ & ++ & - & +++ & +++ \\
\hline rauscheri & ++++ & ++++ & +++ & ++ & - & ++++ & ++ \\
\hline rosinae & +++ & - & - & - & - & - & - \\
\hline schmidi & - & + & $+t$ & - & - & ++++ & + \\
\hline sesverna & + & + & - & - & - & - & - \\
\hline terialensis & ++ & + & ++ & - & - & +++ & - \\
\hline vesulensis & - & - & + & - & - & ++++ & - \\
\hline
\end{tabular}


++ rare, avec moins de $1 \%$ de spécimens

+++ assez abondante, avec 1-5\% de spécimens

++++ abondante, avec $5.20 \%$ de spécimens

+++++ très abondante, avec plus de $20 \%$ de spécimens

En ce qui concerne la dépendance cénotique des espèces (Bodenheimer 1955, Dajoz 1970), on distingue trois catégories:

- Les espèces eucènes ou caractéristiques, qui sont exclusives d'une communauté ou, plus fréquemment, s'y trouvent en plus grand nombre que dans les autres.

- Les espèces tychocènes ou préférantes, qui se rencontrent régulièrement dans plusieurs communautés, tout en préférant l'une d'elles (abondance supérieure).

- Les espèces xénocènes ou étrangères, qui se trouvent accidentellement dans une communauté à laquelle elles n'appartiennent pas.

Brinck (1949), qui disposait de données concernant plus de 400 biotopes, a dressé une classification cénotique très détaillée des Plécoptères de Suède. Comme nous n'avons prospecté qu'un très petit nombre de stations dans la vallée du Pô, nous nous bomerons à étudier la répartition spatiale des espèces recensées en utilisant nos données semiquantitatives. Nous tiendrons compte également de la composition faunistique observée dans plusieurs réseaux hydrographiques des Alpes du Piémont prospectés ces dernières années. Nous ne négligerons pas non plus l'influence particulière de chaque taxon dans sa communauté. En règle générale il n'est pas possible d'évaluer quantitativement le degré de dépendance cénotique des espèces, bien que leur abondance relative nous foumisse des données de comparaison assez valables. Par exemple, en utilisant seulement l'abondance des individus récoltés, on ne peut comparer la dépendance cénotique d'un gros prédateur tel que Perlodes intricatus, avec celle d'une petite espèce à régime alimentaire phytophage telle que Leuctra rauscheri, cohabitant dans le même biotope lotique.

En fonction des critères qui suivent, nous avons établi une classification cénotique des espèces recensées définissant 4 plécoptérocénoses (Tableau VI).

L'étude de la répartition en altitude des espèces a mis en évidence la prédominance de formes orophiles sténothermes d'eau froide dans la partie supérieure, et celle de formes eurythermes dans la partie inférieure du tronçon étudié. La faune de la zone des sources du Pô (station 1) est très net tement différente de celle de son cours aval (station 5). Mais si nous comparons deux stations voisines, l'affinité apparaît assez forte.

Pour préciser le degré d'affinité cénotique des stations étudiées, nous avons calculé les coefficients d'affinitê de Sörensen (Southwood 1968) (Tableau V/A). Toutefois cette méthode ne prend pas en compte le nombre de spécimens récoltés. Sur un deuxième tableau (V/B), les coefficients de Sörensen ont été calculés sans tenir compte des espèces rares et dispersées dans chacune de nos stations (Berthélemy 1966).

La comparaison entre les coefficients des tableaux V/A et V/B fait ressortir deux classifications cénotiques différentes dont celle du tableau V/B semble la plus vraisemblable

Les coefficients du tableau V/A permettent de définir trois communautés de Plécoptères:

1) en altitude, au-dessus de $1.300 \mathrm{~m}$, on trouve une seule communauté composée par la faune des stations 1, 2, 3 et 6 liées par un degré élevé d'affinité cénotique:

2) la station 7 mont te une affinité en moyenne plus marquée pour les stations d'amont que pour les stations d'aval car sa faune composée d'un mélange de formes de différentes valences écologiques, est intermédiaire entre celles des communautés d'amont et d'aval, ce qui suggère de la considérer comme une plécoptérocénose à part ;

3) la communauté de la partie inférieure peut être séparée en deux sous-groupements ; en effet, bien qu'on remarque un assez bon degré d'affinité entre les stations 4 et 5 , la pollution qui affecte cette dernière située à l'altitude la plus basse, appauvrit notablement la faune, ce qui différencie cette station de la précédente.

D'après le tableau V/B, si l'on exclut les espèces rares dans le calcul des coefficients de Sörensen, le degré d'affinité cénotique entre stations adjacentes demeure assez élevé, mais il décroît rapidement pour les stations de plus en plus éloignées et il est donc un peu plus difficile de préciser les plécoptérocenoses. On peut toutefois en distinguer quatre :

1) Plécoptérocénose de l'étage subalpin, au-dessus de $1.900 \mathrm{~m}$ (station 1), dans laquelle prédominent les espèces sténothermes d'eau froide. 
Tableau $V$. Degré d'affinité cénotique des 7 stations.

A : d'après la totalité des espèces recensées. B : d'après toutes les espèces à l'exception des formes rares.

A

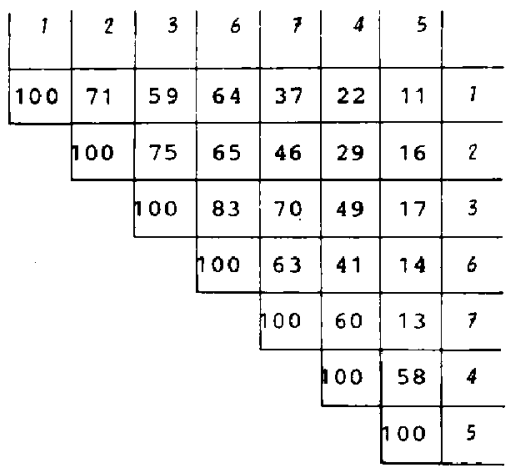

2) Plécoptérocenose de l'étuge montagnard supérieur, entre 1.300 et $1.900 \mathrm{~m}$ (stations 2,3 et 6 ). dans laquelle prédominent encore les espèces sténothermes d'eau froide mais sont également nombreuses les espèces à large valence écologique.

3) Plécoptérocénose intermédiaire de l'étage montagnard inférieur, entre 1.000 et $1.300 \mathrm{~m}$ (station 7), composée d'un mélange d'espèces sténothermes, eury thermes et à large valence écologique.

4) Plécoptérocénose de l'étage submontagnard, entre 400 et $1.000 \mathrm{~m}$ (stations 4 et 5 ), composee par des formes eurythermes et à large valence écologique : elle peut être séparée en deux sous-groupements : a) sous-groupement des cours d'eau peu pollués à fond caillouteux, entre 600 et $1.000 \mathrm{~m}$ (station 4); b) sous-groupement des cours d'eau assez pollués à fond vaseux, entre 400 et $600 \mathrm{~m}$ (station 5).

Nous allons maintenant préciser la composition de chacune de ces communautés.

\subsection{Plécoptérocénose de l'étage subalpin.}

A cette communauté, composée de 20 espèces, dont 7 eucènes, 5 tychocènes et 8 xénocènes (Tableau VI), appartient la seule station 1, c'est-à-dire la zone des sources du Pô. C'est l'abondance des mousses qui entraine la prédominance des
B

\begin{tabular}{|c|c|c|c|c|c|c|c|}
\hline 1 & 2 & 6 & 3 & 7 & 4 & 5 & \\
\hline 100 & 57 & 38 & 33 & 8 & 0 & 0 & 1 \\
\hline & 100 & 61 & 57 & 26 & 11 & 0 & 2 \\
\hline & & 900 & 69 & 43 & 25 & 0 & 6 \\
\hline & & & 100 & 46 & 18 & 0 & 3 \\
\hline & & & & 100 & 67 & 36 & 7 \\
\hline & & & & & 100 & 56 & 4 \\
\hline & & & & & & 100 & 5 \\
\hline
\end{tabular}

Protonentura, tandis que les leuctra, qui se nourrissent surtout de feuilles mortes et de débris végétaux allochtones, peu abondants dans ce biotope, sont représentées par des populations moins nombreuses, à l'exception de la seule $L$. rauscheri. Les prédateurs de la famille des Perlodidae, sont eux aussi des composants typiques de cette communauté grâce à l'abondance de leurs proies habituelles, à savoir les nymphes d'Ephéméroptères et de Plécoptères et les larves de Trichoptères et de Diptères.

Parmi les xénocènes on trouve des formes qui vivent à moyenne et basse altitude : quelques adultes, surtout des femelles ovigères, volent vers la haute vallée, c'est le cas d'Amphinemura sulcicollis, Protonemura nitida et Nemoura sinuata. D'autres, bien qu'orophiles, sont très rares dans ce biotope au-dessus de la limite de la forêt, comme Nemotira mortoni, Leuctra alpina et L. helvetica. Il y a enfin deux espèces strictement a]ticoles, Nemoura oropensis et Leuctra sesvenna, dont nous avons récolté seulement quelques spécimens isolés : la vallée du Pô représente probablement la bordure la plus méridionale de leur aire de répartition.

\subsection{Plécoptérocénose de l'étage montagnard sıpérieur.}

Cette communauté, composée des stations 2,3 tt 6 , est assez semblable à la précédente, mais bien plus 
Tableau VI. Classification cénotique des espèces dans les plécoptérocénoses : $\mathrm{E}=$ eucène, $\mathrm{T}=$ tychocène, $\mathrm{X}=$ xénocène.

\begin{tabular}{|c|c|c|c|c|c|}
\hline \multirow{2}{*}{$\begin{array}{l}\text { plécoptérocénoses de t'écage } \\
\text { altitude an }\end{array}$} & \multirow{2}{*}{$\begin{array}{l}\text { subalpin } \\
>1900\end{array}$} & \multicolumn{2}{|c|}{ poontagnard } & \multicolumn{2}{|c|}{ submon tagnard } \\
\hline & & $1300-1900$ & $1000-1300$ & $600-1000$ & $400-600$ \\
\hline Dictyogenus alpinum & $\mathbf{T}$ & $\mathrm{x}$ & $x$ & - & - \\
\hline Perlodes intricatus & $E$ & $\mathrm{x}$ & - & - & - \\
\hline microcephalus & - & $\mathrm{x}$ & $x$ & - & - \\
\hline Isoperla carbonaria. & - & $x$ & $\mathbf{E}$ & $\mathbf{T}$ & $\mathbf{x}$ \\
\hline grammatica & - & - & - & $\mathrm{x}$ & $\mathrm{x}$. \\
\hline oxylepis & - & - & - & - & $\mathrm{x}$ \\
\hline rivilorum & $\mathrm{E}$ & E & - & - & - \\
\hline Dinocras cephatotes & - & - & $\mathrm{x}$ & $\mathbf{T}$ & - \\
\hline Perla grandis & - & - & $\mathbf{T}$ & - & - \\
\hline marginata & - & $\mathrm{x}$ & - & - & - \\
\hline chlonoperla susemicheli & - & $x$ & - & - & - \\
\hline Siphonaperla montana & $\mathbf{T}$ & $\mathrm{x}$ & - & - & - \\
\hline torrentium & - & $x$ & $\mathrm{x}$ & - & - \\
\hline Rhabdiopteryx alpina & - & $x$ & $x$ & - & - \\
\hline Taencopteryx kühtreiberi & - & $x$ & - & - & - \\
\hline Amphinemura sulcicollis & $\mathrm{x}$ & $\mathrm{x}$ & $\mathbf{E}$ & $\mathrm{E}$ & $\mathbf{E}$ \\
\hline Protonemura ausonia. & - & $x$ & - & - & - \\
\hline brevistyla & $\mathbf{E}$ & $\mathrm{x}$ & - & - & - \\
\hline caprai & - & $x$ & $x$ & - & - \\
\hline intricata & - & - & $\mathrm{T}$ & $\mathbf{T}$ & $\mathrm{T}$ \\
\hline Rateraris & $\mathbf{E}$ & $\mathbf{E}$ & $x$ & - & - \\
\hline nimborella & $\mathbf{E}$ & $\bar{x}$ & - & - & - \\
\hline nimborear & $\mathbf{E}$ & $\mathrm{E}$ & $\mathbf{E}$ & - & - \\
\hline nitida & $\mathrm{x}$ & $\mathrm{E}$ & $\mathrm{T}$ & $\mathbf{T}$ & $\mathrm{x}$ \\
\hline praecox & - & - & - & $x$ & $\mathrm{x}$ \\
\hline Nemoura cinerea selene & - & - & - & $\mathrm{x}$ & $\mathrm{T}$ \\
\hline mortani & $x$ & $\mathrm{~T}$ & $\mathbf{T}$ & - & - \\
\hline oropensis & $x$ & - & - & - & - \\
\hline paleiventris & - & - & - & $x$ & - \\
\hline sinuata & $\mathrm{x}$ & $x$ & $\mathrm{x}$ & - & - \\
\hline uncinata & - & - & $\mathrm{T}$ & $\mathbf{E}$ & $\mathbf{E}$ \\
\hline Nemurella pictetii & - & - & $x$ & - & - \\
\hline Capnia bifrors & - & - & - & - & $x$ \\
\hline rigra & - & $\mathrm{x}$ & - & - & - \\
\hline vidua vidua & - & $T$ & - & $\mathrm{x}$ & - \\
\hline Leuctra alpina & $\mathrm{x}$ & $\mathrm{x}$ & $\mathbf{E}$ & $x$ & - \\
\hline armata & $T$ & $\mathrm{x}$ & $\mathrm{x}$ & - & - \\
\hline elisabethae & - & - & $\mathrm{T}$ & $\mathrm{E}$ & $T$ \\
\hline fusca & - & - & - & $T$ & $\mathrm{x}$ \\
\hline handlirschi & - & - & - & $\mathrm{x}$ & - \\
\hline helvetica & $\mathrm{x}$ & $\mathbf{E}$ & $\mathbf{E}$ & $\mathrm{x}$ & - \\
\hline hippopus & $=$ & - & $\mathrm{x}$ & $\mathrm{E}$ & $\mathbf{E}$ \\
\hline inermis & - & $\mathrm{T}$ & $\mathrm{E}$ & $\mathbf{E}$ & $\mathrm{x}$ \\
\hline leptogaster & - & $\mathrm{x}$ & $\mathrm{x}$ & $x$ & - \\
\hline major & - & $\mathrm{x}$ & $\mathrm{x}$ & $=$ & $=$ \\
\hline meridionalis & - & $\mathrm{x}$ & $\mathrm{x}$ & $\mathrm{E}$ & - \\
\hline moselyi & - & $\mathrm{x}$ & $\mathrm{T}$ & $x$ & - \\
\hline nigra & - & - & - & - & $x$ \\
\hline niveola & - & $\mathrm{x}$ & $\mathrm{E}$ & $\mathrm{T}$ & - \\
\hline rauscheri & $\mathbf{E}$ & $\mathrm{E}$ & $\mathrm{x}$ & $x$ & - \\
\hline rosinae & $\mathbf{T}$ & $=$ & - & - & - \\
\hline schmidi & - & $T$ & $x$ & - & - \\
\hline sesvenna & $\mathrm{x}$ & $x$ & - & - & - \\
\hline teriokensis & $\mathbf{T}$ & $\mathrm{x}$ & - & - & - \\
\hline vesulensis & - & $\mathrm{E}$ & - & - & - \\
\hline
\end{tabular}


diversifiée avec 36 espèces dont 7 eucènes, 4 tychocènes et 25 xénocènes (Tableau VI).

Bien qu'environ les $2 / 3$ des espèces caractéristiques soient communes aux deux plécoptérocénoses, la denivellation de quelques centaines de mètres entre la station 1 et celles situées en aval, suffit à modifier la dépendance cénotique des autres espèces. C'est le cas d'un part de Protonemura brevistyla ef $P$. nimborella, formes strictement orophiles dont les populations s'appauvrissent notablement en dessous de l'étage subalpin, et d'autre part de Protonemura nitida, Nemoura mortoni, Capnia vidua vidua, Le uctra helvetica, L. inermis et $L$ schmidi qui, dans ce secteur des Alpes, deviennent d'autant plus rares que l'altitude est plus élevée. On peut aussi classer parmi les espèces eucènes Leuctra vesulensis, forme sténoendémique de la vallée du Pô, cantonnée dans le ruisseau Martino et très rare dans la station 3 (Ravizza \& Ravizza Dematteis 1984).

Très nombreuses sont les espèces xénocènes, parmi lesquelles on peut distinguer trois groupes:

- Le premier est composé de Siphonoperla montana, Chloroperia susemicheli, Rhabdiopteryx alpina, Taeniopteryx kühtreiberi, Leuctra alpina et $L$. moselyi qui, dans les cours d'eau montagnards du Piémont, sont tantôt eucènes tantôt tychocènes. Il est difficile d'expliquer la rareté de ces espèces dans cette communauté, mais la pollution est sans doute un facteur limitant important. Taeniopteryx kihtreiberi, particulièrement sensible à la pollution, est en voie de disparition dans plusieurs vallées alpines; nous avions récolt tê une exuvie mâle dans la station 3 le 17.IV.1973 et n'avons plus retrouvé cet te espèce dans toute la vallée du Pô pendant les 12 années suivantes.

- Le deuxième groupe est représenté par des espèces orophiles, composantes typiques de la faune subalpine, qui deviennent rares au-dessous de 1.900 $\mathrm{m}$ : Perlodes intricatus, Protonemura brevistyla, $P$. nimborella, Leuctra armata, $L$. sesvema et $L$. teriolensis.

- Le troisième groupe est composé par 13 autres espèces moins sténothermes ou à valence écologique plus large (voir Tableau VI) : en général leurs biotopes préférentiels se situent dans les cours d'eau des étages montagnard inférieur et submontagnard.
9.3. Plécoptérocénose intermédiaire de l'étage montagnard inférieur.

Elle est représentée seulement par la faune de la station 7 comprenant 30 espèces dont 7 eucènes, 7 tychocènes et 16 xénocènes (Tableau VI).

Comme nous l'avons déjà remarqué, cet te communauté montre des affinités avec les plécoptérocénoses adjacentes, toutefois on ne peut pas la rattacher ni à celle d'amont, ni à celle d'aval. En effet parmi les 14 formes eucènes et tychocènes, les espèces à large répartition altitudinale prédominent, mais on rencontre aussi des formes orophiles (Protonemura nimborum et Leuctra helvetica) et des formes de basse altitude (Protonemura intricata, Nemoura uncinata et Lewctra elisabethae).

La richesse et la diversité de cette communauté peuvent être reliées surtout aux facteurs thermiques (amplitude annuelle, fluctuations diumes estivales) et trophiques (abondance des débris végétaux altoch. tones), qui jouent un rôle important dans la composition qualitative et quantitative du peuplement. Cependant on connait encore trop peu cet te communauté et, pour en préciser mieux la composition en Plécoptères et les limites, il faudra étudier d'autres biotopes lotiques de l'étage montagnard inférieur.

\subsection{Plécoptérocénose de l'étage submontagnard.}

Cette communauté comprend les stations du tronçon inférieur la 4 , à $650 \mathrm{~m}$, riche de 25 espèces, et la 5 , à $550 \mathrm{~m}$, qui héberge une faune moins diversifiée avec 15 espèces. C'est le fond vaseux et le degré de pollution plus élevé qui entraînent la réduction importante du nombre des espèces dans cette dernière station par rapport à la précédente. Cet te dif. férence ne permet pas, à notre avis, de considérer les faunes de ces deux stations de façon globale, mais plutôt de préciser deux sous-groupements (Tableau VI).

a) SOUS-GROUPEMENT DES COURS D'EAU PEU POLLUES A FOND CAILLOUTEUX

Il est représenté par la faune de la station 4, com. posée pour la plupart d'especes eurythermes de basse altitude associées à des éléments à large valence écologique. A ce sous-groupement appartiennent les espèces eucènes Amphinemura sulcicollis, Nemoura uncinata, Leuctra elisabethae, L. hippopus, $L$. inermis et $L$. meridionalis, et les espèces tychocènes Isoperla carbonaria, Dinocras cephalotes, 
Protonemura intricata, P. nitida, Leuctra fusca et $L$. niveola. Ce peuplement est typique des cours d'eau submontagnards du versant interne des Alpes occidentales.

\section{b) SOUS GROUPEMENT DES COURS D'EAU ASSEZ POLLUÉS} A FOND VASEUX

La faune de la station 5 (seule composante de ce sous-groupement) n'est représentée que par 15 espèces, dont 12 sont communes avec celles de la station précédente. On peut vraisemblablement supposer que la réduction du peuplement aux scules especes les plus tolérantes à la pollution, est à attribuer au changement des caractéristiques de ce milieu lotique, à cause de l'action directe de l'homme. Toutes les espèces classees parmi les formes eucènes et tychocènes de ce sous-groupement, sont communes et abondantes dans les cours d'eau submontagnards à fond pierreux des Alpes du Piémont et semblent bien s'accommoder aussi des fonds vaseux.

Berthélemy (1966) compare la répartition amontatal des Plécoptères pyrénéens avec la liste des Plécoptères de Tchécoslovaquie rangés en fonction de leurs exigences décroissantes vis-à-vis de la pollution, et il constate une nette similitude entre les deux classements. Les espèces composant la faune de notre station 5 s'inserent bien dans ces classements, cn particulier dans celui des Pyrénées. Naturellemenı le cours submontagnard du Pô ne peut héber. ger ni les formes fluviales qui autrefois habitaient les rivières et les fleuves de plaine et qui sont aujourd'hui en voie de disparition (Ravizza \& Nicolai 1983, Zwick 1984), ni celles qui se cantonnent surtout dans les ruisseaux de basse altitude. Les discordances les plus évidentes se rapportent à deux especes: Perlodes microcephalus et Dinocras cephalotes. La première s'accomode très mal de la pollation (Aubert 1986); dans les Alpes italiennes, elle habite les eaux pures des étages montagnard et submontagnard et est en voie de disparition en dessous de $600-700 \mathrm{~m}$. La seconde est assez abondante par endroits dans les rivières de fond de vallée ou de plaine, méme un peu polluées, pourvu que le fond soit pierreux. A notre connaissance, Dn. cephalote: n'habite pas les cours d'eau à fond vaseux; d'après Berthélemy (1981) elle est absente aussi de ceux dont le fond n'est pas assez stable pour permettre l'installation des mousses.

\section{Conclusion}

Les 55 espèces recensées constituent un peuplement très riche et diversifié. Si nous comparons ce nombre à celui des especes que nous avons récoltées pendant ces douze dernjères années de recherches, dans d'autres cours d'eau des Alpes du Piémont : 45 dans la vallée du Tanaro, 48 dans la val. lée du Grana el 49 dans la vallée d'Oropa (Ravizza \& Ravizza Dematteis 1977, 1986, Ravizza Dematteis \& Ravizza 1983a), nous pouvons supposer que notre inventaire est presque complet.

Les paramètres physico-chimiques de l'eau des stations étudiées sont très semblables; seule la température varie sensiblement en fonction de l'altitude et de l'exposition géographique. C'est donc le régime thermique et, en second lieu, les ressources alimentaires qui apparaissent comme les principaux facteurs de répartition en altitude des espèces. C'est encore la température, dans ses variations annuelles et ses fluctuations journalières, qui cont rôle les périodes d'éclosion et de vol. Dans le Tossiet, l'amplitude thermique diurne entraine l'apparition plus précoce des espèces hivernales, tanctis qu'en altitude, dans le cours de source du Pô, les températures estivales basses prolongent de 3 à 4 mois les pétiodes de vol de quelques espèces orophiles.

Notre recensement a mis en évidence la rareté de Perlodes microcephalus, Isoperla grammatica, Chloroperla susemicheli, Siphonoperla forrentitum, Rhabdiopteryx alpina et Taeniopleryx kiihtreiberi, espèces à répartition extensivic dans les cours d'eau montagnards ou submontagnards des Alpes, et qui sont en général communes et souvent abondantes. 1 l est difficile d'expliquer cette rareté autrement que par la pollution qui affecte plus particulièrement le cours de montagne du Pô. Bien que ce degré de pollution soit peu élevé par rapport à celui des fleuves et des rivières de plaine, la présence en l'eau courante d'insecticides, de produits détergents et de déversement d'égouts, a entraîné de façon inéluctable la raréfaction et parfois la disparition des formes les plus sensibles telles que celles déjà citées.

Parmi les trois espèces les plus intéressantes du point de vue biogéographique: Isoperla oxylepis, Protonemura ausonia et Letictra vesulensis, la première, récoltée seulement dans le secteur le plus pollué du Pô (station 5), est très probablement en voie de disparition, tandis que les deux autres, 
cantonnées dans le ruisseau Martino, ne semblent pour le moment pas en danger.

L'analyse de la répartition des espèces fait ressortir la présence d'au moins quatre plécoptérocénoses principalement liées à l'altitude. Les cours d'cau de la partie supérieure abritent deux communattés sténothermes d'eau froide dans lesquelles prédominent des éléments à répartition alpine associés à des espèces médioeuropéennes montagnardes. Le cours submontagnard du Pô héberge une communauté eurytherme dans laquelle prédominent des éléments à vaste répartition en Europe ou sudeuropéens. La faune du Tossiet, seul ruisseau prospecté dans l'étage montagnard inférieur, appartient à une communauté de transition entre celles des parts supérieures et inférieures du tronçon étudié. Les limites de cette plécoptérocénose, du fait de l'hétérogénéité de ses composantes, sont encore difficiles à préciser.

\section{Remerciements}

Nous exprimons notre plus sincè re reconnaissance à $\mathbf{M}$. le Professeur Charles Degrange, de la Faculté des Sciences de Grenoble, qui a bien voulu faire une lecture critique de notre manuscrit. Nous remercions aussi, bien cordialement, M. le Dr Gilles Vinçon (Grenoble), dont l'aide précieuse nous a permis d'améliorer la forme française de notre note.

\section{Travaux ditcs}

Aubert (J.). 1953. Plécoptères européens nouveaux. Mitt. schweiz. ent. Ges., $26: 72-76$.

A ubert (J.) 1954. Note sur quelques Plécoptères du Piérnont et de Ligurie avec la description de deux espèces nouvelles. Bofl. Soc. ent, it., 84:107-113.

Aubert (J.) 1957. Les Letct ra du groupe de inermis Kempny et quelques espèces inermes isolées (Plécoptères Leuctridae). Mitt. schweiz. ent. Ges., $30: 285-312$.

Aubert (J.) 1959. Plecoptera. In Irsecta Helvetica, Fauna I, Lausanne, $140 \mathrm{p}$.

Aubert (J,) 1986. Les Plécoptères des Alpes françaises. Annls. Soc. ent, Fr, $22: 81-104$.

Berthélemy (C.) 1966 . Recherches écologiques et biogéographiques sur les Plécoptè res et Coléoptères d'eau courante (Hydraena et Elminthidaej des Pyrénées. Annls. Limnol., 2 : 227458.

Berthélemy (C.) 1981. Régimes alimentaires et pièces buccales de quelques Perlodidae et Perlidae des Pyrénées. Annls. Limnol., $17: 1-24$.

Bodenheimer (F.S.) 1955. Précis d'écologie animale. Payot, Paris, $315 \mathrm{p}$.

Brinck (P.) 1949. Studies on Swedish stoneflies (Plecoptera). Opusc. ent. Suppl, $11: 1-250$

Consiglio (C.) 1967. Lista dei Plecotteri della regione italiana. Fragm. ent. $5: 1-64$
Consigljo (C.) 1976. La distribuzione dei Plecotteri italiani. Lav. Soc. it. Biogeogr., N.S., $6: 383-393$.

Dajoz (R.) 1970. Précis d'écologie Dunod, Paris, 357 p.

Gay (C.) 1982. Les communautés benthiques d'un torrent des Alpes françaises: l'Eau d'Olle (Isère). Trav. Lab. Hydrobiol, 7-31.

Gribaudi (D.) 1966. Piemonte e Val d'Aosta. UTET, Torino, $X+603$ p.

Hutchinson (G.E.) 1957. A treatise on Limnology. Wiley \& Sons, New York, 1 : XIII + 1015.

Hynes (H.B.N.) 1976. Biology of Plecoptera. Anntu. Rev. Enomat., $21: 135-154$

Illies (J.) 1978. Plecoptera. In Limnofauna Europaec, G. Fischer Verlag. Seut tgart 264-273.

IRSA 1977. Indagine sulla qualità delle acque del fiume Po. Quademi IRSA, $32: 1-821$.

Jeannel $\{R$.) 1942. La genèse des faunes terrestres. PUF, Paris, VIII $+512 \mathrm{p}$.

Kamler (E.) 1967. Distribution of Plecoptera and Ephemeroptera in relation to altitude above mean sea level and current speed in mountain waters. Pol. Arch. Hrdrobiol., $14: 29-42$.

Lillehammer (A.) 1974. Norwegian stoneflies. II. Distribution and relationship to the environment, Norsk ent. Tidsskr., 21: 195-250.

Rauser (J.) 1971. A contribution to the question of the distribution and evolution of plecopterological communities in Europe. Acta faun. ent. Mus. Nat. Pragae, $14: 33-63$.

Ravizza (C.) 1978. Una specie nuova di Plecottero dei monti del Biellese : Leuctra brevipennis n. sp. Redia, 61 : 251-257.

Ravizza (C.) \& Nicolai (P.) 1983. I Plecotteri minacciati di estinzione nella regione italica. Bolt. Soc. ent. it., $115: 70-78$.

Ravizza (C) \& Ravizza Dematteis (E.) 1976. La plecotterofauna dell'alta val Tanaro (Alpi Liguri). Mem. Soc. ent. it., $55: 183-215$.

Ravizza (C) \& Ravizza Dematteis (E.) 1980. Nemoura oropensis $\mathbf{n}$. sp., endemica dei monti del Biellese (Alpi Pennine). Natura. 71 : $92-100$

Ravizza (C) \& Ravizza Dematteis (E.) 1982. Dedscrizione della ninfa e geonemia di Lenctra brevipennis Ravizza. Atti. Soc. ital, Sci, nat., $123: 349-352$.

Ravizza (C.) \& Ravizza Dematteis (E.) 1983. Sull'ininterootta presenza di Plecot teri adulti in un ruscello dell'Appennino ligure occidentale Analisi della plecotterocenosi e dei periodi di volo. Redia, 66: 615-634.

Ravizza (C.) \& Ravizza Demat teis (E.) 1984. Leuctra vestalensis, new species from the Westem Italian Alps (Plecoptera : Leuctridae). Aguatic Insects, $6: 41.44$.

Ravizza (C.) \& Ravizza Dematteis (E.) 1986. Les Plécoptères du Grana (Alpes Cottiennes méridionales). Boll. Mus. Sci. nat. Torino, $4: 311-339$.

Ravizza Dematteis (E.) \& Ravizza (C.) 1983a. Osservazioni preliminari sui Plecotteri della valle Oropa (Alpi Pennine). Atti XIII Congr. Naz, if. ent, 311-318.

Ravizza Dematteis (E.) \& Ravizza (C.) 1983b. Elenco dei Plexotteri dell'alta valle del Po. Atti Soc. ital. Sci. nat., $124: 291-294$.

Ravizza Dematteis (E.) \& Ravizza (C.) 1985. Leuctra sesventa Auben e L helvetica Aubert netl'alta valle del Po. Note morfologiche e geonemiche. Boll. Mus. reg. Sci, nat. Torino, $3: 381.388$.

Southwood (T.R.E.) 1968. Ecological methods with particular refe rence to the study of Insect Populations. Methuen \& Co., London, VIII +391

Zwick (P.) 1973. Insecta : Plecoptera Phylogenetisches System und Katalog. Das Tierreich, 94 : XXXП +465.

Zwick (P.) 1980. Plecoptera (Steinfliegen). Handb. Zool, IV (2), 26 ; $1-115$.

Zwick (P.) 1984. Rote Liste der Steinfliegen (Plecoptera). in Rote Liste der gefährdeten in der Bundesrepublik Deutschland Naturschutz. aktuell., 1 : 115-116. 